\title{
Nebrija MediaLab: un valor añadido a la docencia y al desarrollo de competencias
}

\author{
Nicolás Grijalba de la Calle \\ Universidad Nebrija. Madrid \\ ngrijalb@nebrija.es \\ Fernando Toledano Cuervas-Mons \\ Universidad Nebrija. Madrid \\ ftoledan@nebrija.es
}

\begin{abstract}
Resumen:
Como consecuencia de los últimos cambios acontecidos en el panorama de la comunicación y de la educación en la Universidad Nebrija se ha creado un laboratorio de medios, denominado Nebrija MediaLab. Un valor añadido a la docencia que se ha diseñado como un espacio multidisciplinar y de encuentro para los estudiantes, en el que puedan reforzar, fuera del aula, las competencias adquiridas en sus respectivas titulaciones de Comunicación, a través del desarrollo de diversos proyectos. Además de convertirse en un escaparate en el que los alumnos puedan mostrar sus trabajos al mundo profesional.
\end{abstract}

Palabras clave: Comunicación; Laboratorio de medios; Proyectos

\section{Nebrija MediaLab: an added value to teaching and competences development}

\begin{abstract}
:
As a result of the lattest changes in comunication and teaching, Nebrija University has created an innovative media lab in the faculty of comnmunication, called Nebrija MediaLab. This constitutes an added value to teaching as it has been designed as an multidisciplinary and meeting space for the students. Here, out of the clasroom, they can reinforce the acquired competences of their Communication degrees, through the development of several proyects. Furthermore, it is window display that allows students to show their work to the professional world.
\end{abstract}

Key Words: Communication; Media lab; Projects

\section{Referencia normalizada:}

Grijalba de la Calle, N. y Toledano Cuervas-Mons, F. (2014): Nebrija MediaLab: un valor añadido a la docencia y al desarrollo de competencias. Historia y Comunicación Social. Vol. 19. Núm. Especial Febrero. Págs. 733-744.

\section{Sumario:}

1. Introducción. 2. Desarrollo de competencias fuera del aula. 3. Puesta en marcha del Nebrija MediaLab. 3.1. Espacios colaborativos. 4. Proyectos del curso 2013-2014. 4.1. Proyecto Tuenti Móvil. 4.2. Proyecto Festival Jóvenes Tocados por la Publicidad. 5. Conclusiones. 6. Referencias bibliográficas. 


\section{Introducción}

El contenido se ha erigido como el eje en el que gira el cambio del modelo comunicativo y más en concreto, el del mundo de la Publicidad. Las marcas comerciales necesitan impactar y atraer a sus consumidores a través de un contenido de calidad. Por tanto se ha pasado de una estrategia intrusiva, en la que las piezas publicitarias interrumpían los momentos de ocio de los consumidores, a una estrategia de atracción, en la que a los receptores se les proponen contenidos relevantes, generados por las marcas, que compiten de igual a igual con las demás formas de entretenimiento en los momentos de ocio. Por lo que es esencial crear espacios de encuentro (Solana, 2012: 35) con los consumidores en los que las acciones publicitarias deben tener la capacidad de atracción suficiente ya que rivalizan, en las mismas condiciones, con largometrajes, representaciones teatrales o, incluso, partidos de fútbol.

Esta nueva estrategia pasa, primero, por conocer los gustos de los consumidores, verdaderos impulsores de este cambio en la comunicación, saber el por qué van a elegir la oferta creada por una determinada marca comercial. Después pasa por saber enganchar esas preferencias de los receptores con la filosofía e imagen de la marca. A continuación crear un contenido que integre a la perfección estos dos aspectos anteriores y que tenga la calidad suficiente para que los receptores lo elijan en sus momentos de ocio. Y, por último, saber seleccionar los medios de comunicación para comunicar la oferta a los receptores.

El cambio en el panorama comunicativo también ha provocado una nueva clasificación en los medios de comunicación en la que se ha superado, en el mundo de la Publicidad, el binomio de medios convencionales y no convencionales, para pasar a hablar de medios pagados, medios propios y medios ganados o método POEM (Paid, Own, Earned Media), según la filosofía Forrester (Ayestarán, Rangel y Sebastián, 2012: 204). Los medios pagados serían en los que se necesita efectuar un pago para conseguir espacios publicitarios, como pueden ser la televisión, radio o prensa. Los medios propios son aquellos de los que disponen y controlan las organizaciones para comunicarse con sus consumidores, como sus páginas web, blogs o redes sociales. Y los medios ganados en los que una marca comercial se convierte en noticia para los medios de comunicación- la denominada Publicity- o en los que los consumidores opinan sobre los productos de una organización en blogs, comunidades o redes sociales.

Esta nueva revolución tecnológica lógicamente también ha estimulado la creación de nuevas figuras profesionales, lo que ha abierto el abanico de oportunidades a los estudiantes a la hora de incorporarse al mercado laboral.

Asimismo estas transformaciones han estimulado un mayor acercamiento, del que ya existía, entre las disciplinas de Comunicación. La Publicidad cada vez se extiende más hacia los territorios de ficción (García, 2007: 59), tan propios de la Comunicación audiovisual. Ya que las marcas comerciales se convierten en auténticas productoras de piezas audiovisuales, tal y como propone el denominado Branded Content 
(Contenido de marca) en las que sus productos se convierten en protagonistas velados de las historias y no solo tiene presencia, en ocasiones algo forzadas, como ocurría con el Product Placement.

Del mismo modo la Publicidad invade espacios propios del Periodismo, así aparece el Brand Journalism o Periodismo de marca, en el que se desarrolla, por medio de informaciones y opiniones, un tema relacionado con la marca comercial a través de blogs especializados.

Todo ello desemboca, sobre todo, en la formación de los estudiantes, ya que en la Facultad de Ciencias de la Comunicación se han empezado a cambiar los planes de estudios adaptándolos a los nuevos cambios en la comunicación y a los nuevos perfiles profesionales. También en las transformaciones en las aulas, creando espacios que fomenten y faciliten el trabajo en equipo y la creatividad. Hasta culminar en la puesta en marcha del Nebrija MediaLab que permite a los estudiantes reforzar las competencias adquiridas en sus titulaciones, por medio de la realización de proyectos planteados por la propia facultad como proyectos más personales en los que pueden plasmar y reflejar sus inquietudes. Con lo que el laboratorio es un escenario multidisciplinar que fomenta la participación, la creatividad y el trabajo en equipo.

\section{Desarrollo de competencias fuera del aula}

Estos cambios en el mundo de la comunicación han venido de la mano de otros cambios en el terreno educativo, así la formación en competencias y el protagonismo del estudiante en su formación que propone el Espacio Europeo de Educación Superior (EEES) han revolucionado la metodología docente tanto dentro como fuera de las aulas.

Con el objetivo de mejorar la formación y el desarrollo de competencias del estudiante, en la Facultad de Ciencias de la Comunicación de la Universidad Nebrija se ha adoptado la filosofía del learning by doing, (aprender haciendo) en la que los alumnos de nuestras titulaciones, viven su profesión desde el primer momento. Por lo que la puesta en marcha del Nebrija MediaLab ha supuesto un gran apoyo para la docencia y una motivación para los estudiantes para desarrollar y mostrar sus proyectos. Este laboratorio se convierte en otra herramienta más para potenciar el aprendizaje práctico de los estudiantes (Arnau y Martín, 2013).

Este curso 2013-2014 se han puesto en marcha dos proyectos propuestos desde la Jefatura de estudios de la facultad, el proyecto Tuenti Móvil y el Proyecto Festival Jóvenes Tocados por la Publicidad, que se desarrollarán a continuación. Estas propuestas están muy cerca de la metodología educativa del aprendizaje orientado a proyectos, que forma estudiantes proactivos y autónomos que tengan que desarrollar sus competencias comunicativas al formar parte de grupos de trabajo (Lorente, Doblas y Zaldumbide, 2011). 
En ambos proyectos se han creado distintos equipos de trabajo, vinculando a estudiantes de las distintas titulaciones de Comunicación y de todos los cursos. Por lo que tiene un carácter multidisciplinar que pretende explotar los diferentes perfiles que se dan en las áreas de la comunicación y fomentar el trabajo en equipo y la inteligencia emocional. Cada uno de los grupos de trabajo está dirigido por dos estudiantes de último curso fomentando así su capacidad de liderazgo, que son los responsables de que el equipo consiga los objetivos previamente fijados. Esta estructura refuerza, de igual modo, la toma de decisiones, la resolución de problemas y la creatividad.

La demanda del mundo profesional de este tipo de competencias transversales cada vez es mayor, como también existe una convergencia de disciplinas y perfiles de las áreas pertenecientes al ámbito de la comunicación. Por tanto, es esencial para los estudiantes trabajar en proyectos cooperativos que imitan situaciones de trabajo que se encontrarán cuando se incorporen al mundo laboral. (Bonet, 2006).

El laboratorio es un instrumento de ayuda a la docencia y al aprendizaje, fuera de las aulas, porque no solo permite al profesor comprobar cómo se comportan los estudiantes ante escenarios pre-profesionales, en los que deben mostrar las competencias adquiridas. Sino que también les permite reforzar sus propias competencias docentes y seguir apoyando a los estudiantes. Como también se convierte en un apoyo para continuar la acción tutorial en el seguimiento personal de cada alumno. Además, de la misma forma, los estudiantes pueden potenciar en los proyectos en los que participen las competencias adquiridas en su titulación.

Asimismo, aparte de los proyectos propuestos por la facultad, el Nebrija MediaLab anima y motiva a los estudiantes a desarrollar proyectos de carácter más personal lo que alienta su iniciativa, su proactividad y su capacidad de emprendimiento.

\section{Puesta en marcha del Nebrija MediaLab}

Para la Facultad de Ciencias de la Comunicación de la Universidad Nebrija era importante que el nuevo curso 2013-2014 comenzase con el proyecto Nebrija MediaLab a punto. Como su nombre indica el concepto sobre el que se articula este espacio es el de la idea de laboratorio. Un centro vivo, en permanente cambio y apostando por las nuevas tecnologías y herramientas, propiciando espacios colaborativos, como veremos más adelante, para el desarrollo de las ideas y los proyectos de los alumnos. El conocimiento que se adquiere en un laboratorio de ideas se aproxima mucho más a los planteamientos de enseñanza, que desde la implantación de los Grados oficiales, persigue la Universidad.

La puesta en marcha del proyecto Nebrija MediaLab hace palpable la máxima que conforma la nueva enseñanza en nuestra Facultad: favorecer el learning by doing, aprender haciendo, proponer modelos de conocimiento más cercanos a la experiencia práctica sin que por ello pierdan peso el análisis y el pensamiento. Afortunadamente, 
estudios como los de Comunicación propician un acercamiento mayor al terreno empírico tales como el desarrollo de trabajos multimedia, en vídeo, de edición y postproducción, realización y planificación audiovisual, diseño gráfico, etcétera.

Parejo al nacimiento de estos nuevos espacios está el afianzamiento de las nuevas áreas colaborativas virtuales para un mejor tratamiento de las asignaturas. Hablamos de los foros, campus virtuales o wikis, en nuestro caso además contenidos en la plataforma Blackboard Learning. Asimismo se favorece un trasvase de información y de comunicación de todo lo acontecido en Nebrija MediaLab a través de su propio blog (http://blogs.nebrija.es/nebrijamedialab/). El blog servirá de escaparate de todos los proyectos que se incardinen en el Laboratorio, así como contenedor virtual de trabajos destacados de alumnos y ex - alumnos de toda la Facultad de Ciencias de la Comunicación.

De esta forma, los primeros meses del curso 2013-2014 han servido para dar a conocer a los estudiantes dicho proyecto. Era fundamental que desde el principio de curso los alumnos tuviesen información sobre esta iniciativa y, sobre todo, que se sintiesen partícipes de este ideal. Que entendieran que Nebrija MediaLab nace como un espacio desde el que surgirán programas de televisión, piezas audiovisuales, campañas publicitarias, periódicos digitales y un largo etcétera de trabajos orientados a la nueva comunicación.

La puesta en marcha de la idea contemplaba, de esta manera, tres puntos claves para su mejor desarrollo y compresión:

- el conocimiento y uso de los nuevos espacios colaborativos;

- el proyecto Tuenti Móvil;

- y, la XVI edición del Festival Jóvenes Tocados por la Publicidad.

\subsection{Espacios colaborativos}

A nuevas fórmulas, nuevos espacios. Los nuevos modelos educativos enuncian la necesidad de hacer una reflexión mayor sobre la arquitectura de los lugares, y de esta forma aprovechar los espacios como instrumentos puramente didácticos.

Era primordial que Nebrija MediaLab se articulase en torno a espacios remozados, alejados de las viejas estructuras y planteamientos. Los estudiantes matriculados en el presente curso, nada más comenzar sus primeras clases, pudieron observar estos cambios.

Dichas aulas se concentran, principalmente, en el Campus de La Berzosa (Madrid), donde se encuentra la Facultad de Ciencias de la Comunicación (lugar donde se imparten todos los Grados). Sin embargo, mantienen una estrecha relación con otra sede de la universidad, Edificio Fundación Nebrija, situada en pleno centro de la capital, donde se cursan todos los Másteres de la Facultad.

Los nuevos espacios han sido bautizados con nombres claros y concretos. 
De esta forma dichos espacios se conocen como:

- Laboratorio 1 (Edición y Postproducción);

- Laboratorio 2 (Diseño gráfico);

- y Laboratorio 3 (Multimedia).

Todos ellos sustituyen antiguos modelos de aulas a la vieja usanza, con pupitres de corte tradicional inamovibles y dirigidos a la atención completa del docente. El proyecto Nebrija MediaLab ha posibilitado un lavado de cara de los espacios: era incoherente proponer un nuevo modelo educativo si las aulas seguían sujetas a querencias de otros tiempos, y a logística más pensada para el modelo de clase magistral.

\subsubsection{Laboratorio 1}

El primero, el Laboratorio 1, se encuentra dentro de los Estudios de Radio y Televisión de la Facultad. Con la incorporación de este espacio, los Estudios consuman un acabado perfecto, fiel reflejo de lo que podría ser hoy en día una pequeña empresa de comunicación, producción y realización. Junto al Plató de Televisión, con su correspondiente control de realización, y al control de Radio Digital con su propio estudio para desarrollar programas e informativos, se encuentra este laboratorio que lleva el sobrenombre de Laboratorio de Edición y Postproducción. Se trata de un espacio equipado con 19 ordenadores que prestan servicio a todos los estudiantes que quieran realizar trabajos de edición. Desde esta aula, próxima a todo lo que acontece en los Estudios, saldrán diferentes productos audiovisuales pensados para todos los canales hipermedia. Desde cortometrajes, pequeñas piezas de ficción, contenido informativo, así como películas publicitarias o virales. Los ordenadores contienen todos los programas necesarios para la edición digital no lineal: destacan Avid y Pro Tools.

El diseño del aula permite el trabajo en equipo, con suficiente espacio entre monitores, así como la presencia de un profesor y/o becario del Estudio si fuera necesario.

\subsubsection{Laboratorio 2}

Pasamos al Laboratorio 2, ya integrado en el edificio principal de la Facultad. Recibe el nombre también de Laboratorio de Diseño Gráfico.

La nueva aula está diseñada en forma de $\mathrm{U}$, propiciando de esta manera unas clases de aspecto más colaborativo. Contiene 20 iMac, mobiliario movible y ergonómico, una gran mesa de trabajo en el centro del aula y un proyector. Además, buscando la claridad y la visibilidad, el espacio se ha liberado de uno de los muros de carga cambiándolo por un gran cristal transparente, que hace las veces de pizarra, consiguiendo de esta forma contagiar al resto de la Facultad de espíritu colaborativo y emprendedor. Las clases, talleres o proyectos que se llevan a cabo ahora dentro de este espacio son visibles para todos los estudiantes y profesores que pasen por allí; 
la transparencia, una de las virtudes más valoradas por los jóvenes de hoy, se hace constante en estos (nuevos) espacios.

De nuevo, los ordenadores contienen todos los programas necesarios para desarrollar un óptimo trabajo en el campo del diseño gráfico: Ilustrator, Photoshop, InDesign, entre otros.

\subsubsection{Laboratorio 3}

Por último, el tercer espacio es el llamado Laboratorio 3, dedicado al trabajo Multimedia. De idéntico corte que el Laboratorio 2, está equipado con ordenadores y proyector, y una mesa de trabajo en medio del aula. Este espacio está especialmente pensado para desarrollar trabajos relacionados con Internet: redes sociales, blogs, campañas publicitarias virales, presentaciones y pitchings, así como la creación de pequeñas piezas audiovisuales pensadas para plataformas como tablets y/o smartphones.

El equipo de becarios de Nebrija MediaLab, alumnos becados por la Universidad pertenecientes a nuestros Grados, trabajan principalmente desde este espacio: controlan todos los procesos técnico/creativos de la plataforma, y dan a conocer a través de los distintos canales reales y virtuales todos los proyectos que se llevan a cabo.

\section{Proyectos del curso 2013-2014}

Desde la jefatura de estudios, con el apoyo de los coordinadores académicos de las titulaciones de Periodismo, Comunicación Audiovisual y Publicidad y Relaciones Públicas, se han propuesto dos proyectos, que si bien son distintos en esencia, comparten la misma filosofía de fomentar espacios de relación y crear equipos de trabajo formado por estudiantes.

Estos proyectos proceden de la trayectoria docente, volcada al aprendizaje práctico, y de las actividades de extensión universitaria, con un alto protagonismo en nuestra universidad. Podíamos mencionar el Festival de Cortometrajes AdN, el Concurso Periodístico Nipho o el mismo Festival Jóvenes Tocados por la Publicidad que en su decimosexta edición se ha vinculado de manera directa al Nebrija MediaLab. Mientras que el otro proyecto Tuenti Móvil, se ha puesto en marcha este curso por primera vez.

\subsection{Proyecto festival jóvenes tocados por la publicidad}

Este festival se ha convertido en una cita casi obligada para los profesionales publicitarios ya que, como suelen destacar, los premios los reciben de los jóvenes, muchas veces los propios receptores de su comunicación. Por tanto el evento se materializa en un punto de encuentro entre los estudiantes y el mundo profesional. 
Pese a ser un certamen con cierto bagaje, como se ha dicho, este año celebra su decimosexta edición, se ha querido relacionar con el Nebrija MediaLab para proporcionarle mayor carácter multidisciplinar y digital, y de esta manera amoldarse a los cambios que se han producido en el área de la Comunicación, la Publicidad y los que se han generado tras la revolución digital. Por tanto, el festival también ha experimentado y está experimentando una serie de cambios.

El primero de estos cambios es que se ha conseguido, aunque es un festival dedicado a la comunicación comercial, la participación de estudiantes de Periodismo y de Comunicación audiovisual y no solo de Publicidad y RR.PP. como ocurría en ediciones anteriores. Con lo que se produce ese acercamiento entre las tres disciplinas al igual que ocurre en los escenarios laborales.

Si damos un repaso por los distintos departamentos que configuran el organigrama de esta presente edición podemos destacar que, si bien hay departamentos en los que existe una presencia abrumadora de estudiantes de Publicidad y RR.PP, aparecen otros en los que la representación de alumnos de las restantes titulaciones de Comunicación es significativa. Algunos departamentos sintonizan, por sus funciones y su perfil requerido, con las preferencias y competencias de los estudiantes.

Los alumnos de Periodismo han elegido el Departamento de RR.PP., medios sociales y gabinete de prensa, ya que su objetivo es mantener el interés de los medios especializados, como pueden ser revistas del sector o programas de radio sobre publicidad, a través de notas de prensa o diferentes comunicados. Además también dinamizan las redes sociales, ya que en estas últimas ediciones el festival tiene una presencia cada vez más activa. Asimismo actualizan y proporcionan contenido al blog del festival, siendo la publicidad y los jóvenes sus temas a desarrollar, lo que se equipara al Periodismo de marca (Brand Journalism).

Mientras que los estudiantes de Comunicación audiovisual han elegido, por coincidencia entre sus gustos, perfiles y competencias, el Departamento de Creatividad, en el que se escoge la idea, el tono visual y se desarrollan todas las piezas gráficas, digitales y audiovisuales del festival, como pueden ser: vídeos virales, vídeos que se proyectan el día del evento o montajes con los anuncios nominados, making-of, entre otros. De esta manera conectan, sobre todo a través de las distintas piezas de ficción que sirven para anunciar el festival, con la filosofía del Branded Content o Contenido de marca. En este departamento también existe una presencia representativa de alumnos de Publicidad y RR.PP. ya que sus funciones coinciden con uno de los perfiles profesionales más queridos y solicitados, a lo largo de los años, por los estudiantes como es el creativo.

En los restantes departamentos existe una presencia más importante de alumnos de Publicidad y Relaciones Públicas ya que los perfiles demandados concuerdan con sus preferencias y competencias. Así nos encontramos con el departamento de Patrocinio, encargado de conseguir apoyo económico para la realización del evento. El Departamento de Investigación, responsabilizado de organizar las distintas fases de investigación: recuerdo espontáneo, recuerdo sugerido y visionado, que precisarán 
los distintos nominados para las categorías de premios. Cabe destacar que otra de las variaciones que se van a acometer, en esta edición, es la referida al visionado, ya que la votación será online y se podrán visualizar los anuncios en la web del festival.

Conforme a los cambios provocados por la cada vez mayor importancia de la Publicidad digital se ha decidido eliminar el Premio al Tocado digital que reconocía la mejor campaña en Internet porque se ha decidido que a todas las categorías de premios puedan optar anuncios para televisión y para Internet indistintamente. Es decir que las grandes categorías de premios, el Gran Tocado que reconoce la mejor campaña del pasado o el Joven Tocado que premia la mejor campaña dirigida a jóvenes pueda resultar ganadora una pieza de Internet y no como sucedía en las ediciones anteriores sólo de televisión. Lo mismo ocurriría con las categorías técnicas: Tocado a la mejor Idea Creativa, Tocado a la mejor Imagen/Realización, Tocado a la mejor Música, Tocado al mejor Storytelling (mejor historia).

Por tanto al vincularlo con el Nebrija MediaLab el Festival Jóvenes Tocados se ha logrado amoldar a esos cambios digitales que se han producido en el mundo publicitario y ha conseguido lograr una mayor presencia de estudiantes de Periodismo y de Comunicación audiovisual en sus equipos de trabajo lo que le ha dotado de un carácter multidisciplinar todavía mayor.

\subsection{Proyecto tuenti móvil}

Se trata de la propuesta estrella, por novedosa, que va a lanzar Nebrija MediaLab en el presente curso. La red social española, con sede en Madrid, cuyo accionista principal a día de hoy es el gigante de las telecomunicaciones Telefónica, ha virado su estrategia comercial hacia el proyecto Tuenti Móvil. Desde febrero de 2012, la red social ha lanzado su propio operador móvil, especialmente pensado para su público potencial, los jóvenes. Las tarifas están adaptadas a este target, pensadas sobre todo para la descarga de datos y el uso constante de aplicaciones y redes sociales, y no tanto, por consiguiente, para realizar llamadas. De esta forma, Tuenti Móvil se ha convertido en una de las operadoras más competitivas del mercado.

Siendo evidentes, por tanto, de que hoy en día los contenidos son los "reyes del mercado", Tuenti Móvil cerró un acuerdo en el presente año con la Universidad Nebrija para desarrollar nuevos productos que, posteriormente, ofrecerán a sus usuarios. El Proyecto Tuenti Móvil, enmarcado en el Nebrija MediaLab, pretende llevar a cabo durante este curso una serie de ficción realizada enteramente por nuestros alumnos, pensada especialmente para su difusión en nuevas plataformas.

La creación y desarrollo de este producto de ficción depende en todo momento de los estudiantes de la Facultad de Ciencias de la Comunicación, siempre con el apoyo y la supervisión de Tuenti Móvil y de los diferentes profesores que entrarán a formar parte del equipo según se sucedan las diversas fases de construcción de esta serie.

Los estudiantes de todas las titulaciones están voluntariamente implicados en todo el proceso, creando una red de colaboración entre diferentes perfiles. Mientras que 
del rodaje de la serie y su producción, así como del desarrollo de guiones, se encargarán estudiantes del Máster de Dirección y Realización de Series de Ficción junto a alumnos del Grado de Comunicación Audiovisual, los estudiantes de Publicidad y Relaciones Públicas se encargarán del diseño de las campañas publicitarias, anuncios gráficos y toda clase de contenidos virales que consigan posicionar a la serie en las distintas plataformas que existen. Por su parte, los estudiantes del área de Periodismo coordinarán las notas de prensa y dotarán al blog de Nebrija MediaLab de contenido interesante y complementario.

Para conseguir una mayor validez en todo este proceso colaborativo, cercano a la experiencia profesional, los alumnos contarán en todo momento con la ayuda de profesores y becarios, así como de un timming concreto -imprescindible para cualquier producción audiovisual- que posibilitará el trabajo eficaz y controlado de todo el proceso.

Tras la presentación del proyecto en el inicio del curso, la primera fase del Proyecto Tuenti Móvil ya está cerrada. Los primeros meses del primer semestre están sirviendo para conocer cuál será la idea que se desarrolle y de la que se extraigan los guiones. Durante quince días (mes de octubre) los alumnos tuvieron la oportunidad de participar en el Concurso de Ideas de Tuenti Móvil. Con una breve sinopsis y un título provisional, esquematizando la idea y focalizando en estas breves líneas el contenido, diecisiete estudiantes participaron aportando su idea seminal.

Todos los proyectos presentados, en su mayoría, destacaron por su carácter cómico y su acercamiento a los intereses de los jóvenes. De carácter preferiblemente abierto, sin grandes tramas ni extensas subtramas, las sinopsis presentadas descansaban sobre personajes con gran personalidad o situaciones algo delirantes que propiciaban aparentemente- interés y atracción. Sin embargo, de entre todas las ideas presentadas un grupo de profesores de la Facultad hizo un primer descarte dejando atrás los proyectos que no cuadraban con el espíritu más innovador o que se acercaban a modelos narrativos más propios del cine o de la televisión.

Los alumnos seleccionados tras esta primera criba, en total cinco estudiantes, tuvieron que realizar un pitching ante un grupo de profesores de todas las áreas de la Comunicación, simulando de esta forma la metodología del mundo profesional. El pitching, herramienta utilizada por las grandes y pequeñas productoras para conocer la estabilidad y fuerza de una idea, se celebró en el Laboratorio 3. Los alumnos elegidos tuvieron la oportunidad de redondear y dar más información sobre sus proyectos y contestar a todas las dudas que surgieron entre los profesores. Asimismo, la metodología del pitching permite ver con mucha más claridad y acierto si una idea es sólida y aprovechable o, si, sin embargo, estamos ante una historia sin demasiado recorrido ficcional. De esta forma, tras deliberación del jurado, tres estudiantes fueron los elegidos para pasar a la fase final. Las tres ideas ganadoras coinciden en su carácter cómico o puramente de entretenimiento, aunque una de ellas tiene potencial de acercarse al marketing digital y a la publicidad. 
A continuación, cada una de estas ideas será desarrollada por un equipo de alumnos del Máster de Dirección y Realización de Series de Ficción, bajo la tutela del profesor de la asignatura Taller de guion para directores, para una vez confeccionados los primeros borradores, las primeras escenas y la esquematización básica de los personajes ser presentados ante el cliente. El cliente, en este caso Tuenti Móvil, elegirá cuál es la idea que más le gusta, la que más se aproxima a sus intereses y la que, pensando siempre en las nuevas pantallas, se adapta mejor a la nueva forma de consumir historias.

Una vez elegida la historia, las distintas fases de construcción son idénticas a las de cualquier producción de ficción. Los siguientes meses del curso servirán para la producción, realización y postproducción de los diferentes capítulos que se determinen, cobrando especial interés el momento del rodaje. Paralelamente a esta actividad, un equipo de estudiantes de los diferentes Grados, bajo el nombre de Equipo Buzzmedia, se encargará de diseñar making-of, potenciar virales informativos para las redes sociales, alimentar el blog del proyecto con diversos contenidos, etcétera. Pretendemos que el Proyecto Tuenti Móvil se convierta en una experiencia audiovisual cercana a las nuevas fórmulas narrativas, al concepto de lo transmedia.

\section{Conclusiones}

El Nebrija Medialab se quiere convertir en el máximo exponente de la filosofía del aprender haciendo de la Facultad de Ciencias de la Comunicación de la Universidad Nebrija, cuya fórmula se aplica tanto a la docencia como a las actividades de extensión universitaria. A modo de implicaciones pedagógicas y conclusiones podemos señalar que el laboratorio se ha convertido en:

- Un espacio de trabajo de carácter multidisciplinar que refuerza las competencias adquiridas en las titulaciones y fomenta la participación e inquietudes de nuestros estudiantes.

- Un valor añadido a la docencia, ya que permite comprobar, a través de una actividad real, cómo han aprendido los alumnos. Además de convertirse en un complemento a la acción tutorial, ya que se puede realizar un mayor seguimiento a nivel personal y de ayuda a cada uno de los estudiantes que participan en los proyectos.

- Un escaparate donde los alumnos pueden enseñar sus mejores trabajos al mundo profesional de cara a su incorporación laboral y que también sirve a la Universidad Nebrija como herramienta de difusión.

- Una simulación del mundo profesional, en el que los alumnos a través de los proyectos, experimentarán los problemas y desafíos con los que se enfrentan los profesionales en su día a día. 


\section{Bibliografía}

\subsection{Libros:}

AYESTARÁN, R. RANGEL, C. y SEBASTIÁN, A. (2012). Planificación estratégica y gestión de la Publicidad. Conectando con el consumidor. Madrid: Esic Editorial.

GARCÍA LÓPEZ, C. (2008). El libro de Bob: la nueva publicidad del siglo XXI. Madrid: Index Book.

SOLANA, D. (2010). Postpublicidad. Reflexiones sobre una cultura publicitaria en la era digital. Barcelona: Postpublicidad.

6.2 Artículos en publicaciones web:

ARNAU, R. y MARTÍN NÚÑEZ, M. (2013). “Aplicaciones digitales online para la gestión de recursos docentes audiovisuales: el modelo del Labcom UJI". En: Estudios sobre el Mensaje Periodístico. Vol. 19 Núm. especial marzo. [30-10-2013].

BONET, M. (2006.). "Centralidad de la comunicación audiovisual en el etorno digital: propuesta desde la experiencia formativa". En: Revista de Universidad y Sociedad del Conocimiento. Vol. 3 - No 2. Octubre. [30-10-2013].

LORENTE, J., DOBLAS, P. y ZALDUMBIDE, I. (2011). "La competencia en comunicación en el aprendizaje orientado a proyectos (PBL)". En: Actas de Univest 2011. Girona. [30-10-2013].

\section{Los autores}

Nicolás Grijalba de la Calle. Licenciado en Ciencias de la Información (Periodismo) por la Universidad Antonio de Nebrija. Doctorando por la Universidad Complutense de Madrid con la tesis "La imagen de Madrid en el cine español". Experiencia en distintos medios como Servimedia, La Gaceta, TVE y en revistas culturales y de tendencias. Compagina la docencia con la participación en congresos, seminarios y talleres, en proyectos informativos y culturales y como organizador de eventos y promotor cultural. Conocimientos de interpretación y dramatización en escuelas de teatro como la Cuarta Pared (Madrid) y presentación de festivales. Entre las últimas propuestas escénicas destacan la colaboración en el proyecto 'Coser y cantar' (Museo Reina Sofía), 'S.A.E'. (Festival Visible 2010) y 'Anatomía Queer' (Espacio Escénico DT, 2011). Actualmente es Coordinador académico de Grado en Comunicación audiovisual de la Universidad Nebrija.

Fernando Toledano Cuervas-Mons. Doctor en Ciencias de la Información por la Universidad Complutense de Madrid con la tesis "El diseño del anuncio gráfico publicitario en medios convencionales: un análisis del método de trabajo del Director de arte" (2010). Máster en Publicidad por ICADE (Universidad Comillas). Licenciado en Ciencias de la Información por la UCM, especialidad en Comunicación Audio- 
visual. Ha trabajado en Gardoqui-Gold, Publicis y RdA Comunicación. También es profesor en el Máster Universitario en Dirección de Publicidad Integrada TBWA de la Universidad Nebrija y ha sido docente en el Curso de Creatividad Publicitaria y de la Comunicación del IED (Istituto Europeo di Design). Pertenece al Comité Ejecutivo del Capítulo académico de la IAA (International Advertising Association). En la actualidad es Coordinador académico de Grado en Publicidad y Relaciones Públicas de la Universidad Nebrija. 